\title{
TCF7L2 polymorphisms are associated with type 2 diabetes in northern Sweden
}

\author{
Sofia Mayans ${ }^{1}$, Kurt Lackovic ${ }^{1}$, Petter Lindgren ${ }^{1}$, Karin Ruikka ${ }^{2}$, Åsa Ågren ${ }^{3}$, Mats Eliasson ${ }^{2,3}$ \\ and Dan Holmberg*,1
}

\author{
${ }^{1}$ Division of Medical and Clinical Genetics, Department of Medical Biosciences, Umea University, Umea, Sweden; \\ ${ }^{2}$ Department of Medicine, Sunderby Hospital, Lulea, Sweden; ${ }^{3}$ Department of Public Health and Clinical Medicine, \\ Umea University, Umea, Sweden
}

A recent study found association of one microsatellite and five single nucleotide polymorphisms (SNPs) in intron 3 of the TCF7L2 gene with type 2 diabetes (T2D) in the Icelandic, Danish and American populations. The aim of the present study was to investigate if those SNPs were associated to T2D in two (familyand population-based) cohorts from northern Sweden. We genotyped four of the associated SNPs in a case-control cohort consisting of 872 T2D cases and 857 controls matched with respect to age, sex and geographical origin and in a sample of 59 extended families (148 affected and 83 unaffected individuals). Here, we report replication of association between T2D and three SNPs in the case-control (rs7901695, $P=0.003 ;$ rs7901346, $P=0.00002 ;$ and rs12255372, $P=0.000004)$ and two SNPs in the family-based (rs7901695, $P=0.01$ and rs7901346, $P=0.04$ ) samples from northern Sweden. This replication strengthens the evidence for involvement of TCF7L2 in T2D.

European Journal of Human Genetics (2007) 15, 342-346. doi:10.1038/sj.ejhg.5201773; published online 24 January 2007

Keywords: association; diabetes; polymorphism; TCF7L2

\section{Introduction}

Type 2 diabetes (T2D) is a multifactorial disorder characterized by chronic hyperglycaemia resulting from pancreatic dysfunction and insulin resistance. It is a common disease with a complex pattern of inheritance, which most likely reflects the influence of multiple genetic and environmental factors. ${ }^{1,2}$ Publication of several genomewide scans has linked many loci to the development of T2D in different populations. ${ }^{3-5}$ Genes implicated in T2D have conferred modest risk ${ }^{6-9}$ and replication efforts have sometimes been successful; ${ }^{10,11}$ however, in many cases they have yielded inconsistent results. ${ }^{8,12}$ We have previously reported linkage of T2D to chromosome 2 in

${ }^{*}$ Correspondence: Dr D Holmberg, Division of Medical and Clinical Genetics, Department of Medical Biosciences, Umeå University, SE90185 Umeå, Sweden.

Tel: + 469078527 02; Fax +4690785 35 93;

E-mail: dan.holmberg@medbio.umu.se

Received 30 August 2006; revised 30 November 2006; accepted 1 December 2006; published online 24 January 2007 families from northern Sweden ${ }^{12}$ and in the same study found weak linkage to 10q. A recent publication found association of one microsatellite and five single nucleotide polymorphisms (SNPs) in intron 3 of the transcription factor 7-like two gene (TCF7L2), localized to chromosome 10q, with T2D in the Icelandic, Danish and American populations. ${ }^{7}$ Moreover, recent functional data linked alleles in some of those SNPs to progression from a state of impaired glucose tolerance (IGT) to T2D. ${ }^{13}$

Based on this background, we set out to investigate if four SNPs in TCF7L2, which had been previously associated to $\mathrm{T} 2 \mathrm{D},{ }^{7}$ were associated to $\mathrm{T} 2 \mathrm{D}$ in both case-control and family-based samples from northern Sweden.

\section{Materials and methods}

Subjects

Both the family-based and the matched case-control samples were as described previously. ${ }^{12}$ Briefly, the familybased material consisted of 231 samples from 59 extended 
families (148 affected and 83 unaffected individuals), and the case-control material comprised 872 T2D cases and 857 controls matched with respect to age, sex and geographical origin. Diabetes diagnoses were confirmed by scrutinizing medical records regarding symptoms and blood glucose measurements, following 1999 World Health Organisation criteria. ${ }^{14}$ Individuals with fasting plasma glucose $>7.0 \mathrm{mmol} / \mathrm{l}$ and 2 -h oral glucose tolerance test (OGTT) plasma glucose $>7.8 \mathrm{mmol} / \mathrm{l}$ but $<10 \mathrm{mmol} / \mathrm{l}$ were considered as having IGT and they were classified as affected. The average BMI was 30.2 for both familial and case-control cases, 26.9 for unaffected familial members, and 25.7 for controls in the case-control cohort. Average age at diagnosis for the cases in the family study was 50 years and average C-peptide was $1520 \mathrm{pmol} / \mathrm{l}$. Average C-peptide for the controls in the family study was $770 \mathrm{pmol} / \mathrm{l}$. Informed consent was obtained from all participants, and the study was carried out with the approval of the regional ethical review board. Genomic DNA was prepared as described previously. ${ }^{12}$

\section{Genotyping of polymorphisms in TCF $7 \mathrm{~L} 2$}

Four SNPs in intron 3 of the TCF7L2 gene, rs7901695, rs7903146, rs11196205 and rs12255372 were genotyped using TaqMan Assay-on-Demand (Applied Biosystems, Foster City, CA, USA). The position of each SNP on chromosome 10 is given in Table 3. Assays were performed according to the manufacturer's instructions. Genotypes were analysed using the allelic discrimination function of the TaqMan 7900HT Fast Real-Time PCR system (Applied Biosystems, Foster City, CA, USA). Five percent of all samples were genotyped in duplicate for each SNP under investigation. Genotyping concordance was found to be $100 \%$ for each SNP. The genotyping success rate was $93.7 \%, 95.4 \%, 93.1 \%$ and $93.7 \%$ for rs7901695, rs7903146, rs11196205 and rs12255372, respectively. The investigated SNPs are located in one haplotype block. Both $D^{\prime}$ and $r^{2}$ values, calculated with Haploview, ${ }^{15}$ are given for the case-control material in Table 1 . As the family-based material is derived from the same population as the casecontrol material, $D^{\prime}$ and $r^{2}$ values should be similar for both materials. Distributions of alleles in the investigated SNPs did not deviate significantly from Hardy-Weinberg equilibrium in controls.

\section{Blood glucose measurements}

After fasting for a minimum of $12 \mathrm{~h}$, blood glucose levels were determined for all healthy individuals in the family and the case-control cohorts using standard laboratory procedures. Next, $75 \mathrm{~g}$ of glucose was given orally and blood glucose measurements were determined $120 \mathrm{~min}$ later.

\section{Statistical analysis}

For testing association in the family-based material, we used a generalized transmission/disequilibrium test (TDT) within the program Transmit, ${ }^{16}$ which allows for missing parental genotypes and multiple affected family members. To compensate for the bias introduced by the fact that the variance may be incorrectly estimated in the presence of linkage, the robust variance estimator was used. ${ }^{16,17}$ In the case-control material, association analysis was performed by means of univariate logistic regression in the software package SPSS 14.0 (SPSS, Chicago, IL, USA). Genotypebased odds ratios (ORs) were calculated using individuals homozygous for the most common allele as the reference. Allele-specific ORs were calculated under the assumption of a multiplicative risk model by assigning 0-2 (depending on each individual's number of at-risk alleles) to a trend variable. To adjust for possible confounders, a fixed effect stratification variable based on geographical area, age and sex was included in the regression model. Three area and three age categories were used for the stratification. A total of 27 strata were used and the effect of the stratification variables was not significant. Haplotype association analysis and estimation of linkage disequilibrium (LD) measurements based on the EM-algorithm were performed in Haploview. ${ }^{15}$ Differences in blood glucose between the genotypes were examined in SPSS 14.0 (SPSS, Chicago, IL, USA) by Kruskal-Wallis test. All statistical tests performed were two-tailed.

\section{Results}

We genotyped four of the associated SNPs, as according to the HapMap, ${ }^{18}$ the fifth SNP (rs7895340) was tagged by one of the other four SNPs (rs11196205), in intron 3 of the TCF7L2 gene, in a case-control material consisting of 872 cases and 857 controls matched for age, sex and geographical origin. Significant association was seen for

Table 1 Linkage disequilibrium between SNPs under investigation

\begin{tabular}{lcccc}
\hline & rs7091695 & rs7903146 & rs11196205 & rs12255372 \\
\hline rs7091695 & & 1.00 & 0.97 & 0.90 \\
rs7903146 & 0.90 & 0.97 & 0.91 \\
rs 11196205 & 0.47 & 0.42 & 0.42 & 1.00 \\
rs12255372 & 0.68 & 0.77 & \\
\hline
\end{tabular}

$\mathrm{D}^{\prime}$ values are represented in the upper right region $r^{2}$ values in the lower left. 
three of the four SNPs, rs7901695 $(P=0.003)$, rs7903146 $(P=0.00003)$ and rs12255372 $(P=0.00002)$, conferring ORs of $1.28,1.42$ and 1.47 , respectively (Table 2$). P$-values presented are from the multiplicative model described in the statistical section. The same four SNPs were then genotyped in 231 samples from 59 families (117 patients with T2D, 12 with diabetic OGTT, 19 patients with IGT and 83 unaffected adults). Association was seen for two of the four SNPs, rs7901695 $(P=0.005)$ and rs7903146 $(P=0.01)$ (Table 3).

For the three SNPs associated in the case-control material, heterozygous individuals clearly conferred greater risk compared to non-carriers $(P<0.05$ for each SNP, Table 2), enabling rejection of a recessive mode of inheritance. However, homozygous individuals did not confer significantly increased risk compared to heterozygous carriers $(P>0.05$ for each SNP, data not shown), allowing for both dominant and multiplicative modes of inheritance in our case-control material.

Blood glucose measurements were performed for all healthy individuals from both the multiplex families and the case-control cohort. The healthy individuals from the families were analysed based on their rs7903146 and rs7901695 genotypes, since these were the SNPs that were associated in the family material. A tendency towards higher blood glucose levels, both fasting and 120 min after challenge with $75 \mathrm{~g}$ of glucose, was observed in individuals homozygous for the susceptible allele (fasting $5.4 \mathrm{mmol} / \mathrm{l}$, glucose challenge $6.3 \mathrm{mmol} / \mathrm{l}$ ) compared to individuals homozygous for the protective allele (fasting $5.0 \mathrm{mmol} / \mathrm{l}$, glucose challenge $5.2 \mathrm{mmol} / \mathrm{l}$ ) (Table 4 ). In both cases, for both SNPs, heterozygous individuals (fasting $4.7 \mathrm{mmol} / \mathrm{l}$, glucose challenge $5.2 \mathrm{mmol} / \mathrm{l}$ ) behaved more like the individuals homozygous for the protective allele. The healthy individuals from the case-control cohort were analysed based on their rs7903146, rs7901695 and rs12255372 genotypes. No difference in either fasting blood glucose levels or levels after glucose challenge could be found based on any of the genotypes (Table 4).

\section{Discussion}

We have successfully replicated the previously found association $^{7,13}$ between SNPs in TCF7L2 and T2D. Three of the SNPs were associated to disease in a case-control material and two of these SNPs were associated to T2D in a family-based material. Individuals in both materials originate from northern Sweden. In contrast to Grant et al. ${ }^{7}$

Table 3 Association of SNPs in intron 3 of TCF7L2 to T2D in families from northern Sweden

\begin{tabular}{lcccc}
\hline SNP & Position $^{\mathrm{a}}$ & Risk allele & Frequency & P-value \\
\hline rs7901695 & 114418675 & $\mathrm{C}$ & 0.24 & 0.005 \\
rs7903146 & 114422936 & $\mathrm{~T}$ & 0.22 & 0.01 \\
rs11196205 & 114471634 & $\mathrm{C}$ & 0.37 & 0.2 \\
rs12255372 & 114473489 & $\mathrm{~T}$ & 0.20 & 0.1 \\
\hline
\end{tabular}

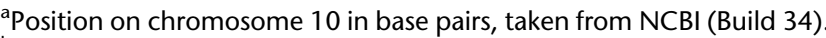

${ }^{b} 1$ degree of freedom.

Table 2 Association of SNPs in intron 3 of TCF7L2 to type 2 diabetes in a case-control material from northern Sweden

\begin{tabular}{|c|c|c|c|c|}
\hline & No. of cases (\%) & No. of controls (\%) & OR $(95 \% \mathrm{Cl})$ & P-value \\
\hline$\pi$ & $434(52.6)$ & $469(59.1)$ & Ref. & \\
\hline Multiplicative model & & & $1.28(1.09-1.51)$ & 0.003 \\
\hline \multicolumn{5}{|l|}{ rs7903146 } \\
\hline$\pi$ & $54(6.6)$ & $35(4.3)$ & $1.85(1.18-2.90)$ & 0.007 \\
\hline Multiplicative model & & & $1.42(1.21-1.69)$ & $3 \times 10^{-5}$ \\
\hline \multicolumn{5}{|l|}{ rs11196205 } \\
\hline GG & $275(33.6)$ & $282(35.8)$ & Ref. & \\
\hline CG & $412(50.4)$ & $388(49.3)$ & $1.08(0.87-1.34)$ & 0.5 \\
\hline $\mathrm{CC}$ & $131(16.0)$ & 117 (14.9) & $1.15(0.85-1.56)$ & 0.4 \\
\hline \multicolumn{5}{|l|}{ rs12255372 } \\
\hline$\pi$ & $38(4.6)$ & $28(3.6)$ & $1.56(0.94-2.59)$ & 0.09 \\
\hline Multiplicative model & & & $1.47(1.23-1.76)$ & $2 \times 10$ \\
\hline
\end{tabular}


Table 4 Blood glucose levels in healthy individuals from the family material and the case-control cohort

\begin{tabular}{|c|c|c|c|c|c|c|c|c|}
\hline & \multicolumn{4}{|c|}{ Fasting blood glucose $(\mathrm{mmol} / \mathrm{l})$} & \multicolumn{4}{|c|}{ Blood glucose at $2 \mathrm{~h}$ OGTT $(\mathrm{mmol} / \mathrm{l})$} \\
\hline & \multicolumn{2}{|c|}{ Family- based material } & \multicolumn{2}{|c|}{ Case-control cohort } & \multicolumn{2}{|c|}{ Family-based material } & \multicolumn{2}{|c|}{ Case-control cohort } \\
\hline & Average $\pm S D$ & P-value & Average $\pm S D$ & P-value & Average $\pm S D$ & P-value & Average $\pm S D$ & P-value \\
\hline rs 7901695 & & 0.15 & & 0.11 & & 0.21 & & 0.85 \\
\hline$\pi$ & $5.0 \pm 0.6$ & & $5.2 \pm 0.7$ & & $5.2 \pm 1.0$ & & $6.5 \pm 1.6$ & \\
\hline CT & $4.7 \pm 0.7$ & & $5.2 \pm 1.0$ & & $5.2 \pm 0.9$ & & $6.5 \pm 1.5$ & \\
\hline CC & $5.4 \pm 0.1$ & & $53 \pm 0.7$ & & $6.3 \pm 0.8$ & & $6.4 \pm 1.3$ & \\
\hline rs790146 & & 0.17 & & 0.16 & & 0.16 & & 0.66 \\
\hline CC & $5.0 \pm 0.6$ & & $5.2 \pm 0.7$ & & $5.2 \pm 0.9$ & & $6.5 \pm 1.7$ & \\
\hline CT & $4.7 \pm 0.7$ & & $5.3 \pm 1.0$ & & $5.2 \pm 0.9$ & & $6.5 \pm 1.5$ & \\
\hline$\pi$ & $5.4 \pm 0.1$ & & $5.4 \pm 0.7$ & & $6.3 \pm 0.8$ & & $6.4 \pm 1.3$ & \\
\hline rs12255372 & & & & 0.15 & & & & 0.48 \\
\hline GG & & & $5.2 \pm 0.7$ & & & & $6.5 \pm 1.7$ & \\
\hline GT & & & $5.3 \pm 1.0$ & & & & $6.6 \pm 1.4$ & \\
\hline$\pi$ & & & $5.4 \pm 0.7$ & & & & $6.4 \pm 1.2$ & \\
\hline
\end{tabular}

Data are means \pm SD. Blood glucose was measured at fasting and $2 \mathrm{~h}$ after an OGTT.

we could not find association of SNP rs11196205 in either our case-control or family-based material, possibly due to lower power in our smaller sample size and/or founder effects resulting in reduced genetic diversity in northern Sweden, compared to Iceland, Denmark and America. Founder effects would suggest that the non-associated polymorphism arose after the disease causing mutation. Furthermore, the fact that three SNPs were associated in the case-control material, but only two of those SNPs were associated in the family-based material, might be due to the fact that the family-based material has a lower power of detecting association than the case-control material. Another possibility is reduced genetic diversity within the families under investigation, even though both the case-control and family-based materials are derived from the northern Swedish population.

Our ORs for rs7901695, rs7903146 and rs12255372 (1.28, 1.42 and 1.47 , respectively) are similar to the relative risks (RRs) determined by combining data from the Icelandic, Danish and American populations for the same three SNPs $\left(1.49,1.54\right.$ and 1.52 , respectively) ${ }^{7}$

Analysis of inheritance models of the SNPs investigated revealed the possibility for both dominant and multiplicative modes of inheritance in our case-control material. The possibility of dominant inheritance could be rejected by Grant et al. ${ }^{7}$ who analysed a microsatellite (DG10S478) in intron 3 of TCF7L2 using a larger casecontrol material. Blood glucose measurements from healthy family members indicate that variation in TCF7L2 affects the individual's capacity to tolerate glucose. However, as only three individuals with the disease susceptible allele in rs7903146 or rs7901695 were healthy, it is difficult to obtain statistical significance between the genotype groups. Analysis of the controls in the case-control cohort resulted in no difference in the capacity to tolerate glucose in any of the SNPs analysed. Measurements of BMI, blood pressure, triglycerides and cholesterol did not show any difference between the SNP genotypes of interest. Further studies are necessary in order to identify more healthy individuals with the disease susceptible genotypes, and to test other parameters such as insulin secretion, in order to elucidate how variations in TCF7L2 influence the pathogenesis of T2D.

Several recent studies have investigated the effect of SNPs in TCF7L 2 on T2D development. Florez et al. ${ }^{13}$ investigated the role of rs12255372 and rs7903146 on the transition from IGT to T2D. They found that individuals homozygous for the $\mathrm{T}$ allele of rs7903146 were more likely to progress from a state of IGT to T2D than individuals homozygous for the $\mathrm{C}$ allele. Cauchi et al. ${ }^{19}$ found that the $\mathrm{T}$ allele of rs7903146 predicts hyperglycaemia in a non-selected, prospectively followed, French population and they found association between rs7903146, rs12255372 and T2D in the French population. ${ }^{20}$ Saxena et al. ${ }^{21}$ found that individuals homozygous for the rs7903146 risk allele had a significant reduction in insulinogenic index and insulin disposition index (DI). Damcott et al. ${ }^{22}$ found significant association with insulin sensitivity index, acute insulin response to glucose and DI in non-Amish Caucasian subjects. These results indicate a possible functional role for SNPs in TCF7L2 and suggest that TCF7L2 has a role in regulation of genes involved in insulin sensitivity and glucosestimulated insulin release.

Expression of the proglucagon gene is transcriptionally regulated by the $\beta$-catenin/TCF protein complex in gut endocrine L cells, but not in pancreatic islet $\alpha$ cells. $^{23}$ Variants of TCF7L2 could therefore, alter levels of glucagon-like protein-1 (GLP-1), an insulinotropic hormone encoded by the proglucagon gene. As GLP-1 works in concert with insulin to help maintain blood glucose 
homeostasis, ${ }^{23}$ altered levels of GLP-1 could very well influence susceptibility to T2D.

By testing glucose homeostasis in healthy individuals genotyped for the T2D-associated polymorphisms in TCF $7 L 2$, a greater understanding of such an influence could be obtained. Further studies are necessary in order to identify more unaffected individuals with the disease susceptible genotypes, and to test other parameters such as insulin secretion, in order to elucidate how variations in TCF7L2 influence the pathogenesis of T2D.

\section{Acknowledgements}

This work was supported by grants from The Swedish Research Council-M, The Kempe Foundation, Norrbotten County Council and the Joint Committee of County Councils in northern Sweden. We are very grateful to all participants in this study. The authors are not aware of any duality of interest.

\section{References}

1 Malecki MT: Genetics of type 2 diabetes mellitus. Diabetes Res Clin Pract 2005; 68 (Suppl 1): S10-S21.

2 Parikh H, Groop L: Candidate genes for type 2 diabetes. Rev Endocr Metab Disord 2004; 5: 151-176.

3 Hanis CL, Boerwinkle E, Chakraborty R et al: A genome-wide search for human non-insulin-dependent (type 2) diabetes genes reveals a major susceptibility locus on chromosome 2. Nat Genet 1996; 13: 161-166.

4 Mahtani MM, Widen E, Lehto $\mathrm{M}$ et al: Mapping of a gene for type 2 diabetes associated with an insulin secretion defect by a genome scan in Finnish families. Nat Genet 1996; 14: 90-94.

5 Elbein SC, Hoffman MD, Teng K, Leppert MF, Hasstedt SJ: A genome-wide search for type 2 diabetes susceptibility genes in Utah Caucasians. Diabetes 1999; 48: 1175 - 1182.

6 Horikawa Y, Oda N, Cox NJ et al: Genetic variation in the gene encoding calpain-10 is associated with type 2 diabetes mellitus. Nat Genet 2000; 26: 163-175.

7 Grant SF, Thorleifsson G, Reynisdottir I et al: Variant of transcription factor 7-like 2 (TCF7L2) gene confers risk of type 2 diabetes. Nat Genet 2006; 38: 320-323.

8 Altshuler D, Hirschhorn JN, Klannemark M et al: The common PPARgamma Pro12Ala polymorphism is associated with decreased risk of type 2 diabetes. Nat Genet 2000; 26: 76-80.

9 Hani EH, Boutin P, Durand E et al: Missense mutations in the pancreatic islet beta cell inwardly rectifying $\mathrm{K}+$ channel gene (KIR6.2/BIR): a meta-analysis suggests a role in the polygenic basis of type II diabetes mellitus in Caucasians. Diabetologia 1998; 41: $1511-1515$
10 Gloyn AL, Weedon MN, Owen KR et al: Large-scale association studies of variants in genes encoding the pancreatic beta-cell KATP channel subunits Kir6.2 (KCNJ11) and SUR1 (ABCC8) confirm that the KCNJ11 E23 K variant is associated with type 2 diabetes. Diabetes 2003; 52: 568-572.

11 Gloyn AL, Hashim Y, Ashcroft SJ, Ashfield R, Wiltshire S, Turner RC: Association studies of variants in promoter and coding regions of beta-cell ATP-sensitive K-channel genes SUR1 and Kir6.2 with type 2 diabetes mellitus (UKPDS 53). Diabet Med 2001; 18: 206-212.

12 Einarsdottir E, Mayans S, Ruikka $\mathrm{K}$ et al: Linkage but not association of calpain-10 to type 2 diabetes replicated in northern Sweden. Diabetes 2006; 55: 1879-1883.

13 Florez JC, Jablonski KA, Bayley N et al: TCF7L2 polymorphisms and progression to diabetes in the Diabetes Prevention Program. N Engl J Med 2006; 355: 241-250.

14 WHO: Definition, Diagnosis and Classification of Diabetes Mellitus and its Complications: Report of a WHO consultation. Geneva: World Health Organization, 1999; 1-59.

15 Barrett JC, Fry B, Maller J, Daly MJ: Haploview: analysis and visualization of LD and haplotype maps. Bioinformatics 2005; 21: 263-265.

16 Clayton D: A generalization of the transmission/disequilibrium test for uncertain-haplotype transmission. Am J Hum Genet 1999; 65: $1170-1177$

17 Huber P: The behaviour of maximum likelihood estimates under nonstandard conditions: Proceedings of the Fifth Berkeley Symposium in Mathematical Statistics and Probability. Berkeley: University of California Press, 1967; 1: pp. 221-233.

18 Altshuler D, Brooks LD, Chakravarti A, Collins FS, Daly MJ, Donnelly P: A haplotype map of the human genome. Nature 2005; 437: 1299-1320.

19 Cauchi S, Meyre D, Choquet $\mathrm{H}$ et al: TCF7L2 variation predicts hyperglycemia incidence in a French general population: the data from an epidemiological study on the insulin resistance syndrome (DESIR) study. Diabetes 2006; 55: 3189-3192.

20 Cauchi S, Meyre D, Dina C et al: Transcription factor TCF7L2 genetic study in the French population: expression in human beta-cells and adipose tissue and strong association with type 2 diabetes. Diabetes 2006; 55: 2903-2908.

21 Saxena R, Gianniny L, Burtt NP et al: Common single nucleotide polymorphisms in TCF7L2 are reproducibly associated with type 2 diabetes and reduce the insulin response to glucose in nondiabetic individuals. Diabetes 2006; 55: 2890-2895.

22 Damcott CM, Pollin TI, Reinhart LJ et al: Polymorphisms in the transcription factor 7-like 2 (TCF7L2) gene are associated with type 2 diabetes in the Amish: replication and evidence for a role in both insulin secretion and insulin resistance. Diabetes 2006; 55: $2654-2659$

23 Yi F, Brubaker PL, Jin T: TCF-4 mediates cell type-specific regulation of proglucagon gene expression by beta-catenin and glycogen synthase kinase-3beta. J Biol Chem 2005; 280: $1457-1464$. 